\title{
Vessel Target Location Estimation During the TIPS Procedure
}

\author{
Guillaume Piliere, \\ CASILab, Division of Neurosurgery, University of North Carolina-CH, Chapel Hill, NC 27599 USA \\ Mark H. Van Horn, PhD, \\ CASILab, Division of Neurosurgery, University of North Carolina-CH, Chapel Hill, NC 27599 USA
}

Robert Dixon, MD, Department of Radiology, University of North Carolina-CH, Chapel Hill, NC 27599 USA

Joseph Stavas, MD, Department of Radiology, University of North Carolina-CH, Chapel Hill, NC 27599 USA

Stephen Aylward, PhD, and

Kitware, Clifton Park, NY 12065

Elizabeth Bullitt, MD

CASILab, Division of Neurosurgery, University of North Carolina-CH, Chapel Hill, NC 27599 USA

\begin{abstract}
Creation of a Transjugular Intrahepatic Portosystemic Shunt (TIPS) requires passage of a needle toward a moving target that is only seen transiently by x-ray prior to needle passage. Intraoperative, 3D target localization would facilitate target access and improve the safety of the procedure. The clinical assumption is that patients undergoing the TIPS procedure possess rigid, cirrhotic livers that undergo only intraoperative translation without significant deformation or rotation. Based upon this assumption, we hypothesize that the position of any unseen, 3D target point within the liver can be determined intraoperatively by precalculation of the relative positions of the target point to a different 3D point that can be tracked intraoperatively. This paper examines this hypothesis using intraoperatively acquired, biplane, x-ray images of 7 patients. In 6 , we tracked the effects of cardiac and respiratory motion, and in 3 the effects of needle pressure. Methods involved reconstruction of $3 \mathrm{D}$ vessel bifurcation and other trackable intrahepatic points from biplane angiograms, measurement of liver deformation by examining changing distances between these 3D points over time, and comparison of expected to actual displacements of these points with respect to a fixed reference point in the liver. We conclude that, for the rigid livers associated with patients undergoing TIPS, that there is less intraoperative deformation than previously reported by other groups addressing healthy liver deformation, and that the location of an unseen target can be predicted within $3 \mathrm{~mm}$ accuracy.
\end{abstract}

\section{Index Terms}

Liver deformation; 2D/3D point reconstruction; TIPS; X-Rays

\footnotetext{
(C) 2009 Elsevier B.V. All rights reserved.
}

Publisher's Disclaimer: This is a PDF file of an unedited manuscript that has been accepted for publication. As a service to our customers we are providing this early version of the manuscript. The manuscript will undergo copyediting, typesetting, and review of the resulting proof before it is published in its final citable form. Please note that during the production process errors may be discovered which could affect the content, and all legal disclaimers that apply to the journal pertain. 


\section{Introduction}

This paper examines the hypothesis that patients undergoing Transjugular Intrahepatic Portosystemic Shunt (TIPS) placement have rigid, nondeformable livers that do not significantly rotate or deform intraoperatively. If this hypothesis is true it would enable the development of 3D guidance applications for this and other hepatic procedures.

The purpose of the TIPS procedure is to reduce portal hypertension (increased pressure within the portal veins supplying the liver). Portal hypertension may result from a variety of disease processes. In industrialized nations it is most commonly secondary to cirrhosis, which in turn is most commonly secondary to alcohol abuse or viral hepatitis (Blei, 2007; Bromley and Kaufman, 2004). The rigid, fibrotic livers associated with these diseases do not permit the normal drainage of blood that enters the liver via the portal vein, thus raising the pressure within the portal vein. Multiple complications may result from such portal hypertension, including ascites (abdominal fluid), variceal hemorrhage (bleeding from enlarged veins), congestive splenomegaly (enlargement of the spleen), hepatorenal syndrome (kidney failure), hepatic hydrothorax (fluid in the chest cavity) and hepatopulmonary syndrome (pulmonary failure). These complications are treated by using a combination of medical, endoscopic, surgical and endovascular means, including liver transplantation and TIPS, which can be used as a bridge to transplant.

The goal of the TIPS procedure is to relieve elevated portal venous pressure by inserting a stent within the liver to connect the portal and hepatic veins, allowing the high pressure portal venous system to drain into the hepatic venous system. This drainage decreases the portosystemic pressure gradient, and thereby helps to treat the various complications.

The procedure is performed endovascularly (Figure 1). During the procedure, the interventionalist inserts a catheter into the internal jugular vein in the patient's neck and, under $\mathrm{x}$-ray guidance, advances it along the major veins of the chest and thence into the hepatic vein. A needle is then passed through the liver, from the hepatic vein into the target portal vein. This is the most critical portion of the procedure, and is the step that may result in serious morbidity or mortality. Once stable access to the portal vein is achieved, a metallic stent is deployed to create a permanent conduit between the two venous systems (hepatic and portal), thereby reducing the elevated portal pressure.

Traditionally, the procedure is guided only by fluoroscopy (a stream of projection x-rays). The primary difficulty with the procedure as traditionally performed is that the target portal vein cannot be seen during needle passage. Although the interventionalist's catheter or needle can be seen continuously on fluoroscopic images, the two venous systems cannot. The hepatic venous system can only be visualized transiently via injection of a contrast agent that opacifies the hepatic veins for a few seconds. Worse, the target portal vein can only be visualized via a different and temporally separated injection of $\mathrm{CO}_{2}$, which diffuses through the liver and opacifies the portal system for only a few seconds. In addition, this $\mathrm{CO}_{2}$ portogram frequently results in a suboptimal image. Since the liver moves as a result of respiratory motion, cardiac motion, and needle pressure, the interventionalist's needle is thus directed toward a continuously moving target that cannot be seen.

The advancement of a long, curved, needle through the liver parenchyma and into the portal vein is the most challenging portion of this procedure and is associated with hemorrhagic complications that are potentially fatal. Over the past 2 decades TIPS have been created in thousands of patients worldwide (Boyer and Haskal, 2005), with a complication rate of 1 $30 \%$ (Bromley and Kaufman, 2004). Although direct procedure related mortality is only $1 \%$ (Bromley and Kaufman, 2004; Haskal et al., 2001; Hassoun and Pomier-Layrargues, 2004), potentially significant complications such as transcapsular needle puncture (passing the needle 
outside of the liver) occur in 5-30\% of cases (Bromley and Kaufman, 2004; Boyer and Haskal, 2005).

The clinical assumptions are that the diseased liver in TIPS patients is rigid and that liver motion is constrained by ligaments and the ribcage. As a result, the clinician assumes that there is only translational motion in the rostro-caudal (head-to-foot) direction and that there is little deformation or rotation of the liver (Saxon and Keller, 1997; LaBerge et al., 1993). When passing the needle towards an unseen target, the interventionalist aims for the point at which the target portal venous system was last seen, adding approximately a centimeter in the caudal (towards the foot) direction to allow for translational motion along a single axis. Multiple needle passes are usually required, which may result in extracapsular passes of the needle, extrahepatic portal vein puncture, and potentially significant intraperitoneal bleeding.

Although ultrasound may be employed to aid in navigation, this system is cumbersome and an average of 4 needle passes is still required (Rose et al., 2000).

A better method of three-dimensional (3D) image guidance would be highly desirable. Our clinicians state that any 3D guidance system that continuously displays the clinician's catheter in $3 \mathrm{D}$ and that continuously reports the changing position of the 3D target point within $5 \mathrm{~mm}$ accuracy would be valuable.

Our group is developing an augmented reality, real-time, true 3D display system to aid in endovascular surgical guidance and that uses biplane x-ray views for intraoperative imaging updates (Maupu et al., 2005). A critical step, regardless of the visualization method employed, is rapid and accurate update of the 3D target position under conditions in which the target may be continuously moving but cannot be seen by intraoperative x-ray. This paper examines two related questions using intraoperatively acquired, biplane, $\mathrm{x}$-ray images. The first is the clinical assumption that the liver in patients with liver failure does not deform. The second is how accurately an unseen 3D target point within the liver can be tracked given a known initial relationship to a different $3 \mathrm{D}$, intrahepatic point that can be tracked continuously by biplane $\mathrm{x}$-ray. The paper is organized to provide the methods employed, the results obtained, and a final discussion of the results.

\section{METHODS}

\section{A. Image acquisition, $x$-ray calibration, and 3D point reconstruction}

At our institution, the TIPS procedure is traditionally performed under monoplane or biplane $\mathrm{x}$-ray guidance (Siemens Neurostar, 10-bit grayscale, 1024×1024 pixel resolution). We henceforth refer to the two approximately orthogonal views obtained during biplane imaging as anteroposterior (AP) and lateral. Our methods for 3D image guidance require biplane acquisition.

Our approach reconstructs 3D points from their projections on biplane x-ray images. This step requires calibration of the two $x$-ray systems. For system calibration, we employ a 3D phantom containing multiple spherical metallic targets encased within a rigid Plexiglas framework. The projections of these targets can be seen clearly on each x-ray view. The relative locations of each target in the designed physical phantom are known. Once the approximately orthogonal $\mathrm{x}$-ray sources have been calibrated, a 3D point whose projections are correctly associated on each projection view can be reconstructed into 3D with an average error of less than a millimeter (Baert et al., 2003; Faugeras, 1996). Our methods of system calibration and 3D point reconstruction are similar to those employed by the above cited groups. 


\section{B. Clinical protocol and overview of approach}

Following advancement of the catheter into the hepatic venous system, a contrast-filled balloon is wedged into a branch of the hepatic vein. This balloon can thereafter be recognized on continuously acquired $\mathrm{x}$-ray views without need of further contrast injection. The center of this balloon provides a point within the liver that moves as the liver moves with respiration, cardiac motion, and needle pressure. Consent for balloon insertion was obtained from all patients involved in this study since insertion of such a balloon is not included in the usual clinical protocol.

During the procedure, the interventionalist normally injects contrast to visualize the hepatic vasculature and, at a different time, $\mathrm{CO}_{2}$ to visualize the portal venous system. Given the methods described above, the 3D relationship between the balloon and a vascular branch point (whose paired projections can be identified on biplane views) can be determined at this moment in time. Similarly, the 3D relationship between the balloon and any other radiopaque object within the liver (e.g., a metallic clip left as a result of prior surgery or a radiopaque gallstone) can also be determined at this moment in time.

If a second set of biplane views is acquired at a later time and displays the balloon and the same set of points, it is possible to perform multiple comparisons. First, one can examine the distances between reconstructed 3D points at times 1 and 2 . Such comparison provides an estimate of liver deformation-in the absence of deformation, the distances between all 3D points should remain constant at times 1 and 2. Second, one can compare the 3D location of a reconstructed 3D "target" point relative to the $3 \mathrm{D}$ balloon at time 1 and time 2 . Such comparison provides an estimate of the ability to track an unseen target point relative to the trackable balloon. Figure 2 illustrates our approach using an illustration of a single projection view at times 1 and 2. Please note that our study employed 3D calculations rather than the simplified pair of 2D illustration portrayed in Figure 2.

\section{Patient selection}

This paper analyzes intraoperative images of 7 patients, each of whom underwent biplane imaging of the same portion of the vasculature or of the same intrahepatic radiopaque objects at two moments in time.

We separate the report of our results into two categories dependent upon the intraoperative image data available: the effects of respiratory and cardiac motion (6 patients) and the effects of needle pressure ( 3 patients). For patients whose respiratory and cardiac motions were assessed, images were acquired either as a continuous biplane video during a breathing sequence or as two separated snapshots during a procedure in which the phase during the breathing cycle was unknown. For each patient, we analyzed the two pairs of biplane images that provided maximum displacement of the balloon between times 1 and 2. This study contains all 6 patients imaged at two time points and in whom at least two indentifiable 3D structures (vessel branch points, surgical clips, gallstones, a stent point) could be indentified with certainty For the 3 patients studied during needle passage, the images represented a continuous biplane video during needle pass. Only 3 patients underwent this biplane imaging procedure and so only 3 patients were studied in this fashion. Respiratory and cardiac motion may have contributed to additional displacement of intrahepatic structures during this needle pass.

The 7 patients included in this paper represent all patients whose images were acquired over a 5 year period and for whom good quality biplane images of the same intrahepatic structures were obtained during at least two moments in time. It should be stressed that the inclusion criteria for the images employed in the current study were highly stringent since we required identification of 3D points whose paired projections could be unequivocally identified on AP 
and lateral projection views at two time points and often under differing imaging circumstances (contrast injection versus $\mathrm{C} 02$ administration, for example). The patient population studied was, as best we can tell, a representative sample of the patients who undergo TIPS at our institution except that a larger than usual number possessed intrinsic radio-opaque objects that could be identified on biplane x-ray views. These objects included radio-opaque gallstones, metallic clips within the gall bladder, and a previously inserted vascular stent. Our proposed technique of surgical guidance does not require the presence of such objects.

\section{Point determination}

For each patient, we identified all intrahepatic points whose paired AP and lateral projections could be unequivocally defined on AP and lateral views at both times 1 and 2 .

Since it can be difficult to associate paired point projections on orthogonal views, we employed the principles of epipolar geometry and the 2D-3D registration of a patient-specific vascular model segmented from a computed tomographic scan of the same patient. Our methods of 3D vessel segmentation and of 3D-2D registration have previously been described in the literature (Aylward and Bullitt, 2002; Pathak et al., 2005) and are not further discussed here. For each intrahepatic 3D point whose associated projections could be defined on biplane images at each of the two times, the point was reconstructed into $3 \mathrm{D}$ using the techniques described above.

Three different types of intrahepatic points were analyzed. The first type comprised vessel branchpoints whose paired projections could be identified on each x-ray view at both time points (11 3D branchpoints). The second type comprised identifiable points along the stent following stent insertion and as studied during a breathing sequence (6 3D stent points). The third type included radio-opaque structures within the liver ( 3 opacified gall stones and 1 surgical clip).

More specifically, the study of respiratory and cardiac motion included 6 patients. For patients 1 and 2, we identified 3-4 vessel branchpoints per sequence of images for a total of eleven 3D branchpoints (two separate sequences were available for patient 2). For patients 3, 4 and 5 it was not possible to determine vessel branchpoints conclusively because of extensive overlap of vessels on projection. Patients 3, 4 and 5 provided respiratory series that included both the balloon and the radiopaque intrahepatic stent itself, however. For patients 3, 4 and 5 we therefore picked two identifiable, intrahepatic points on each stent: the first an opaque marker on the stent and the second the stent midpoint, to provide a total of $63 \mathrm{D}$ stent points that could be tracked over time. Patients 2 and 5 also had a partially opacified gall bladder as a result of gallstones, and one to two identifiable points were selected for each patient for an additional 3 3D points. Patient 6 did not have either an implanted balloon or identifiable vessel branchpoints. We include this patient, however, because visual inspection of biplane images during a breathing sequence suggested deformation of the stent with respiration. This stent was furthermore unusual because it contained identifiable metallic markers along its length. We therefore examined deformation of this stent during respiration by assessing the potentially changing distance between three identifiable points along the stent itself.

Acquiring appropriate images for the study of the effects of needle passage is even more difficult. Since the vasculature cannot be seen during needle insertion, vessel branchpoints cannot be used to assess liver deformation. Since the stent has not yet been inserted, the stent itself cannot be assessed for bending. Analysis is therefore dependent upon radiopaque structures within the liver itself. This study includes 3 patients with intrinsic, intrahepatic radiopaque markers who underwent biplane imaging during needle passage and who also had an implanted intravascular balloon. One patient (patient 7) possessed metallic clips as a result of prior surgery; one identifiable clip could be selected on each of the biplane views and reconstructed into 3D. A second patient (patient 2) had a gallstone that could be similarly 
recognized on biplane views and reconstructed into 3D. The third patient (patient 5) had a partially opacified gallbladder as a result of gallstones, and two identifiable 3D points could be selected with a separation of approximately $1 \mathrm{~cm}$. These two 3D points were the same as the intrinsic liver markers analyzed for patient 5 during respiratory motion.

\section{E. Quantitative studies: Respiration and cardiac motion}

This paper discusses 3 quantitative studies related to the effects of respiratory and cardiac motion in 6 patients.

1) Measurement of balloon excursion between times 1 and 2-For each patient, we measured the 3D distance the balloon moved between time 1 and time 2 . This measurement is useful when interpreting the significance of the deformation measurements or the difference between predicted and calculated vessel branch point or stent point positions.

2) Estimation of the extent of liver deformation-In the presence of liver deformation between times 1 and 2, the distances between identified 3D points lying within the liver will change between times 1 and 2 . As a means of estimating the amount of deformation of the liver during respiration we therefore measured the 3D distances between all identifiable, intrahepatic, 3D points (balloon, branchpoints, stentpoints, clips, stent points) at both times 1 and 2 . The mean, standard deviation and maximum values of the differences of distances between 3D points at times 1 and 2 are given for each imaged subject under Results.

\section{3) Accuracy of predicting the location of an unseen target-The single most} important question examined in this paper was how accurately the location of an unseen target point within the liver (e.g., a target point within the portal venous system) can be predicted given the ability to track the 3D position of a single, different intrahepatic point (e.g., an opacified balloon wedged into the vasculature). If the liver undergoes only translation intraoperatively, the location of an unseen target point can be correctly determined at time 2 given a) an initial 3D vector calculated at time 1 with this vector providing both the direction and the distance between the target and a trackable second point (balloon), and b) the translation of the balloon at time 2. If, however, the liver undergoes either significant rotation or deformation between times 1 and 2 it will not be possible to calculate the location of an unseen target point at time 2 by measuring the displacement of a second, single, trackable point. Please note that the following study does not attempt to distinguish between rotation and deformation. Both deformation and rotation, either alone or in combination, can confound any prediction of point displacements whose calculations are based upon the assumptions that the organ in question is rigid and undergoes translational motion alone.

For all non-balloon points reconstructed into 3D at time 1 we calculated a 3D vector relating that 3D point to the balloon's center. At time 2, each point's predicted location was calculated by adding the original $3 \mathrm{D}$ vector to the new $3 \mathrm{D}$ location of the balloon. This value was then compared to each 3D point's calculated location at time 2 as determined from its reconstruction into 3D from the biplane images obtained at time 2 .

This paper compares the predicted and calculated 3D locations of each non-balloon point at time 2. For each patient, we calculated the mean, standard deviation, and maximum of the 3D distance between predicted and calculated point locations at time 2 .

\section{F. Quantitative studies: Effects of needle pressure}

This paper discusses 3 quantitative studies related to the effects of needle pressure in 3 patients who had a) biplane video sequences obtained during needle push, b) earlier insertion of a balloon wedged into the hepatic vasculature, and, c) at least one intrinsic, radiopaque point 
within the liver that could be reconstructed into 3D from its biplane projections. We do not include an evaluation of liver deformation during this study because there were too few intrinsic, radiopaque points within the liver to evaluate more than the expected and actual locations of a single, intrinsic, radiopaque, liver point relative to the position of the balloon.

1) Measurement of balloon excursion-Measurement of balloon excursion was done in the same fashion as for patients evaluated for respiratory and cardiac motion.

2) Measurement of needle push distance-The $3 \mathrm{D}$ distance that the needle tip traversed during the video sequence of needle push was calculated for each patient. This distance was calculated by manually defining the needle tip on each biplane view and reconstructing its position into $3 \mathrm{D}$ at times 1 and 2 . It should be expected that the needle push distance should be greater than that of balloon excursion, since the needle penetrates the liver and the balloon moves with the liver as the liver is displaced. We included calculation of needle push distance to provide a context within which other point displacements could be interpreted.

3) Accuracy of predicting the location of an unseen target-Comparison of the predicted versus the calculated position of each radio-opaque intrahepatic point was calculated in the same way as in the study of respiration.

\section{G. Analysis of sources of error}

There are at least 4 analyzable sources of error present in this study.

First, the reconstruction of 3D points from pairs of biplane views is subject to error if the initial system calibration is incorrect. Similar to the approach employed by other groups, we employ for system calibration a 3D phantom containing multiple small metallic balls, as outlined above. The relative positions of the balls in a tooled physical phantom are known with certainty. It is thus possible to assess the errors inherent to system calibration by picking an identifiable, central point within the phantom as the origin and reconstructing that point into $3 \mathrm{D}$ from its projections on the calibrated biplane views. The known, physical distances between each of the other metal balls and the ball chosen as the phantom's origin can then be compared to the calculated distances when each ball (including the one representing the origin) is reconstructed into 3D from its biplane projections. Under Results, we present the differences between the calculated and the known, physical distances from the origin for 29 metal spheres in a physical phantom imaged during a TIPS procedure.

Second, there is inherent uncertainty in defining 2D projection points from noisy $\mathrm{x}$-ray images due to projection overlap. For this study, all 2D projection points (balloon center, vessel branchpoints, gallbladder points, and stent points) were determined manually. This manual process is both painful and time-consuming. For the current study, a single individual (GP) performed all manual 2D point selections. In order to assess the reproducibility of his choices, he repeated the process four times (twice during day 1 and twice during day 2, with a 24 hour interval between trials). Under Results, we provide for each patient the mean and standard deviation of the distance between the averaged 3D point positions and the four 3D points used to derive the averaged $3 \mathrm{D}$ point. These data provide an indication of the precision with which $3 \mathrm{D}$ points can be determined from our $\mathrm{x}$-ray images when the same $3 \mathrm{D}$ projection points are selected by the same user within a 48 hour period.

A third potential source of error results from the fact that our AP and lateral images were not acquired exactly simultaneously. Our study employed AP and lateral biplane views acquired at 3 biplane views/second, and with a variable time delay of about 0.2 seconds between each $\mathrm{AP}$ and lateral view acquisition. We attempted to estimate the effects of this delay by evaluating the images of a patient whose balloon translated $1 \mathrm{~cm}$ in 3D space over a period of 5 seconds. 
The differences in calculated 3D locations of the balloon were assessed when each AP image was held constant but the two sequential lateral images, each bracketing the time point at which the AP view was actually acquired, were employed for 3D point reconstruction. Although this approach provides a falsely high estimate of the actual error induced by non-simultaneous view acquisition, it does provide an upper bound of such error.

Finally, the 3D distances between our picked points and the balloon could affect the results of this study. If all picked points were within a few $\mathrm{mm}$ of the balloon, for example, our tracking results might not be applicable to the actual clinical target, generally located 1-2 centimeters away from the balloon. We therefore calculated the distance between the balloon and each of the $3 \mathrm{D}$ picked points, assessing both the range and the percentage of $3 \mathrm{D}$ points located more than $50 \mathrm{~mm}$ from the balloon center-a distance likely to far exceed that of the actual clinical target from the balloon.

\section{RESULTS}

\section{A. Effects of respiratory and cardiac motion}

Figure 3 provides an example of AP and lateral views obtained from the same patient at times 1 and 2 and in whom vessel branchpoints were used to assess liver motion. Note the obvious displacement of the balloon as a result of respiratory and cardiac motion. Note also the associated displacement of a flagged vessel branchpoint. Although additional branchpoints were obtained from the image shown, only one is illustrated here for purposes of clarity.

Figure 4 illustrates AP and lateral views of a stent with radiopaque markers along the length of a catheter that is positioned within the stent. Note the displacement of both the balloon and the stent at time 2 . These images also provide an example of an opacified gall bladder that similarly moves between times 1 and 2 .

Table 1 provides a summary of the 3 quantitative studies performed on liver motion as a result of heartbeat and respiratory motion. The precision of 3D point calculation, to be discussed later, is additionally given in this table.

The calculated 3D balloon excursion between times 1 and 2 ranged from a minimum of about $2.5 \mathrm{~mm}$ (patient 3 ) to a maximum of about $16 \mathrm{~mm}$ (patient 2). These distances represent the translation of a single tracked point within the liver under the combined effects of respiration and cardiac motion which may induce liver translation, rotation, and/or deformation in unknown combination and degree.

The deformation study indicated an average change in the calculated distances between all 3D points at times 1 and 2 of less than a millimeter. The maximum change in distance between $3 \mathrm{D}$ points for all seven patients was less than $3 \mathrm{~mm}$.

The difference at time 2 between predicted and calculated 3D point locations was 1-2 mm. There were no obvious differences between analyses of vessel branch points or stent points. The maximum discrepancy between predicted and calculated values for all points analyzed was less than $3 \mathrm{~mm}$.

\section{B. Combined effects of needle pressure, respiration, and cardiac motion}

Figure 5 illustrates the positions of the needle, the balloon, and an intrahepatic radiopaque point at times 1 and 2 for a patient with clips. As previously noted, needle push was performed rapidly and over much less than a full respiratory cycle. Visual inspection of the video sequences for all 3 patients suggested that the radiopaque points always moved in concert with the balloon. By contrast, the needle, whose motion initially seemed to displace the balloon and the 
radiopaque object together, always moved a much greater distance than the balloon and other objects of interest since the needle penetrated the liver.

Table 2 provides a summary of the 3 quantitative studies performed on the effects of needle pressure as well as the results of precision of $3 \mathrm{D}$ point calculation.

Balloon excursion ranged from somewhat over $1 \mathrm{~mm}$ to somewhat over $7 \mathrm{~mm}$. By contrast, the tip of the needle moved from 19 to over $50 \mathrm{~mm}$ during needle push. The difference between the predicted and the calculated locations of the opacified liver points was approximately 1 $\mathrm{mm}$, with a maximum error of $2.12 \mathrm{~mm}$. This amount of error in the difference between predicted and calculated values is within our clinicians' request for less than $5 \mathrm{~mm}$ error when providing 3D image guidance.

\section{Analysis of contributing errors}

Liver deformation and/or rotation are not the only possible sources of error when calculating the locations of 3D points from their x-ray projections. We have attempted to estimate the contributions of four additional potential sources of error.

Errors as a result of camera miscalibration can certainly affect the results. Following system calibration, we thus compared the known to the calculated distances between the phantom's origin and 29 metal spheres in a machine tooled, 3D physical phantom of known configuration. Results are here expressed in millimeters and in the coordinate system of the camera acquiring the AP view. Errors in the reconstructed distances were respectively in $\mathrm{x}, \mathrm{y}$, and $\mathrm{z} 0.25+0.54$, $0.07+0.51$, and $0.3+0.70 \mathrm{~mm}$, with maximum errors of $1.30,1.30$, and $1.73 \mathrm{~mm}$. The largest errors were present with spheres imaged at the image periphery. It seems likely that image distortion, more pronounced at the periphery of an image, was a contributing factor. It should be noted that the region of interest to the radiologist is generally positioned at the center of the image rather than at the periphery, however. Our average error in 3D distance calculation was submillimeter, as is consistent with the reports of others using similar methods (Baert et al., 2003; Faugeras, 1996). It therefore does not seem likely that camera miscalibration was a single major source of error.

A second source of potential error is in the manual selection of paired projection points. As shown by Table 1 and Table 2, the repeatability of selecting the same paired points when performed by a single operator within a 48 hour period resulted in submillimeter reproduction of the same 3D point.

A third source of potential error arises from the approximately 0.2 second delay between the acquisition of each AP view and its associated lateral view. When an object is moving rapidly during biplane image acquisition, the fact that the biplane images are not acquired exactly simultaneously may produce errors in $3 \mathrm{D}$ point reconstruction. For each AP image, there is no lateral image acquired with the same time stamp as the AP view. One must instead perform $3 \mathrm{D}$ point reconstruction using a lateral view acquired either immediately before or immediately after the AP view in question. When using the same AP view and reconstructing a 3D point twice, the first time using the lateral view acquired immediately before and the second the lateral view acquired immediately after the AP image acquisition, the distance between the two 3D reconstructed points was $1.6 \mathrm{~mm}$ in a patient undergoing rapid liver displacement. If one assumes that the "perfect" lateral view would have been acquired at a time half way between the two times of the actual lateral acquisitions, the error in 3D point reconstruction as a result of non-simultaneous AP and lateral view would be approximately half of the calculated distance between our two calculated points, or about $0.8 \mathrm{~mm}$. 
A fourth source of error might result if most of our 3D picked points were situated very close to the balloon. In such case, it might not be possible to draw conclusions about the ability to relate the balloon to an actual clinical target located 10-20 mm away from the balloon. In the current study, 3D picked points ranged from 18.96 to $140.01 \mathrm{~mm}$ away from the balloon, with a mean distance of $63.03 \mathrm{~mm}$. Indeed, $72 \%$ of the 3D picked points lay at a distance larger than $50 \mathrm{~mm}$ from the balloon - and thus were more distant than any likely clinical target point.

In general, our error analyses suggested that the error resulting from camera miscalibration was approximately $0.6 \mathrm{~mm}$, that there was approximately a $0.3 \mathrm{~mm}$ discrepency when repeatedly selecting paired $2 \mathrm{D}$ points to create a $3 \mathrm{D}$ point, and an error of about $0.8 \mathrm{~mm}$ because the two projection views were not acquired simultaneously. Our 3D point analyses included a majority of points located far more distant from the balloon than the likely target point. Overall, an unseen object within the diseased and rigid livers of patients undergoing the TIPS procedure appears to be trackable within $3 \mathrm{~mm}$ accuracy using biplane projection views.

\section{DISCUSSION}

The greatest challenge of the TIPS procedure is passage of a needle through the liver towards a target that can only be seen at an earlier time point and under conditions in which respiratory motion, heartbeat, and the passage of the needle itself may displace the target. As previously noted, the complication rate of the procedure is in the range of $1-30 \%$, with the majority of serious hemorrhagic complications associated with needle mispuncture (Bromley and Kaufman, 2004; Boyer and Haskal, 2005; Banares et al., 1998). Our clinicians have stated that any type of real-time, 3D image guidance that can provide a continuous update of target location within $5 \mathrm{~mm}$ accuracy during needle passage would be of value.

A number of groups have evaluated displacement of the healthy liver with respiration, concluding that the liver moves approximately $10-15 \mathrm{~mm}$ during the respiratory cycle (Clifford et al., 2002; King et al., 2001; Tokuda et al., 2006). Motion is predominantly in the rostrocaudal (head to foot) direction, but movement of several millimeters occurs along the other two axes as well (Venkatraman et al., 2004). Cardiac motion contributes less to liver motion than does respiration, but cardiac motion may produce displacements of 1- 2 millimeters predominantly in the lateral direction (Venkatraman et al., 2004).

Our paper does not attempt to separate between respiratory and cardiac motion but rather analyzes the two forces together. Our results are consistent with earlier studies. The time required for needle push is brief and occupies much less than a full respiratory cycle. Dependent upon both the sharpness of the needle and the initial resistance provided by the liver surface, liver displacement during needle pressure (as measured by balloon excursion) may vary from equal to that produced during respiration to a value much less than this, although the needle may penetrate deeply within the liver. Furthermore, given the high procedural complication rate and the variably large displacement of the unseen target, which moves in unknown fashion during needle pressure, our results underscore the significance of deriving better methods of surgical guidance.

A critical unanswered question has been whether the liver undergoes significant deformation and/or rotation during TIPS. If neither significant deformation nor rotation of the liver occurs, then it should be possible to estimate the location of an unseen target using prior knowledge of its position relative to a second 3D point that can be tracked intraoperatively. Several studies have evaluated deformation of the liver in healthy subjects, concluding that deformation as a result of respiratory motion is in the range of $10-15 \mathrm{~mm}$ (Blackall et al., 2001; Rohlfing et al., 2001). Miga and colleagues recently reported a study of intraoperative deformation of the liver 
during exploratory surgery and noted similar amounts of deformation in the range of $10 \mathrm{~mm}$ (Miga et al., 2003).

Our own results suggest a much smaller amount of liver deformation, and are consistent with the clinical assumptions made by interventionalists performing TIPS procedures. Indeed, the maximum difference between calculated and measured locations of 3D points in the current study was less than $3 \mathrm{~mm}$, even when assessing 3D points located many centimeters from the balloon. We believe that the discrepancy between our own and prior studies can be explained by the fact that previous studies have evaluated only patients with healthy livers. By comparison with the deformable livers of healthy patients, the cirrhotic, shrunken livers in patients undergoing TIPS are usually fibrotic and hard (Bosch, 2006; Anand, 1999; Kazemi et al., 2006). It is therefore not clear that the deformation results obtained for healthy subjects are directly relevant to the patient group actually undergoing the TIPS procedure. To our knowledge, the current study is the first to examine liver deformation, rotation, and the effects of needle pressure during actual TIPS operations. It should be noted, however, that the results of the current study are applicable only to patients with generalized liver failure and cannot be extended to operations upon patients with healthy livers or to patients with only focal liver disease.

Our approach to providing better 3D guidance for TIPS procedures is based upon use of the $2 \mathrm{D}$ projection angiograms with which clinicians are accustomed. Other approaches are under development by other groups. Intraoperative 3D ultrasound is one such measure (Rose et al., 2000; Rose et al., 2002), but requires a second clinician to manipulate the ultrasound head at a site remote from the interventionalist and moreover seems to require an average of four needle passes (Rose et al., 2000). A method of hybrid cross-sectional angiography imaging is under development by Frisoli and colleagues (Sze, 2006). The Inria and Harvard groups are jointly exploring use of a percutaneously inserted "anchor needle" into the liver with tracking of the external needle base, but analysis of breathing motion in animal models suggests that this approach does not adequately reflect global liver motion and that errors of less than $5 \mathrm{~mm}$ apply to only about half of the liver parenchyma (Bricault et al., 2005). Cleary and colleagues are developing an electromagnetic navigation method to aid in the correction of respiratory motion during liver biopsy, but experiments in swine have indicated a median distance from the needle tip to the center of the tumor of about $8.3 \mathrm{~mm}$ (Banovac et al., 2005). Robotic systems are also under development for ultrasound-guided liver ablative therapy (Boctor et al., 2004), but the accuracy with which the system can adapt to respiratory and other motion is not yet known. Direct MR-guidance systems have been proposed for TIPS in the past (Kee et al., 1999), but it is difficult to find recent papers on the topic. 3D-DSA can be used to provide an initial description of 3D hepatic vessels (Virmani 2007) with a single circulation delineated at a time, but the acquisition and reconstruction times required (many minutes) preclude use for the type of real-time application required here. Repeated 3D-DSA reconstructions, even of a single circulation, might have been useful in evaluating the primary biplane, balloon tracking methods described in our report However, the majority of commercially available rotational angiography suites are designed for intracranial work and few institutions, including our own, possess units capable of providing high-quality reconstructions of hepatic vasculature.

Moreover, the approach would require repeated contrast loading and irradiation of severely ill patients.

It might also be possible to employ sequential 3D imaging techniques to address deformation during breathing in TIPS patients. As noted above, repeated 3D rotational angiographic studies would probably be the procedure of choice, but would require invasive access to the hepatic arteries and portal veins and involve contrast load and radiation to severely ill patients for solely research purposes. CTA would suffer from similar limitations (contrast load/radiation) and it would be difficult to visualize the target portal vein. MRA and MRV are acquired too slowly 
to permit accurate resolution of vascular structures that move with cardiac and respiratory motion without dual gating, which few groups possess. Volumetric Interpolated Breath-hold Examination (VIBE) MR sequences provide another option, and it would indeed be possible to perform a study in which a patient was brought back twice (or imaged twice during a single sitting) at an unknown stage in the breathing cycle. This approach could provide information about a large number of vessel branchpoints and other features within the liver, but the stage in the breathing cycle would be unknown and no knowledge of the effect of needle push would be available. Accurate comparison of 3D ultrasound images over time is difficult because of the limited coverage and because of difficulties with registration - this is still a research area under investigation by many groups. Since ultrasound does not depict many structures but does define blood vessels, a method for ultrasound registration that is dependent upon vessel registration and branchpoint recognition is currently under development by our own group. However, it would probably not be valid to measure distances between branchpoints as the endpoint when these same branchpoints were used for registration. A disadvantage of all of these methods is that they cannot provide real-time information about what happens during needle push.

The approach described in the current paper requires tracking the needle and balloon on readily available biplane views, reconstructing points and curves into 3D, and subsequently providing a display of arbitrary complexity, all of which can be done in real-time (Maupu et al., 2005). The critical assumption, however, has been that one can track an unseen target point with reasonable accuracy (less than $5 \mathrm{~mm}$ ) given an initial known relationship to a different point that can be tracked continuously during the procedure.

Our conclusion that an unseen point within the liver can be tracked within $3 \mathrm{~mm}$ during both respiration and needle insertion implies that the difference between predicted and calculated 3D point displacement is related to liver deformation and/or rotation. In fact, however, some of this discrepancy may be related to at least five sources of error in our methods themselves. We have attempted to analyze four of these potential sources of error (camera miscalibration, precision of 3D point calculation, the effects of non-simultaneous view acquisition, and distance of 3D picked points from the balloon ) as outlined under our Methods and Results sections. Our results suggest that although none of these potential sources of error is, in isolation, likely to produce large errors in our calculations, the cumulative effects of different sources of error may account for a significant proportion of the total error noted in the current study. Yet a fifth potential source of error is that some of our tracked points lay within the gall bladder rather than within the liver itself. Although the gall bladder is closely associated with the liver and moves with it, it may be capable of slight deformation relative to the liver. We have no means of assessing or correcting for such errors, and so our reported results may actually be slightly worse than the results actually would be when correlating the positions of true intrahepatic points alone.

A reasonable question might be why we did not use 3D ultrasound during this study of intraoperative liver deformation/rotation. There are two answers to this question, the first of which relates to clinical need and the second to technical issues. In terms of clinical need, our study already increased procedural time because of the requirements of assessing the relationship between the two projection views using a physical phantom, of inserting an intravascular balloon not usually required by the clinical procedure, and of performing multiple biplane view acquisitions. Although our goal is ultimately to reduce procedural time and to increase patient safety, we cannot use our methods to guide a procedure until they are proven safe. Performance of the current study therefore already increased intraoperative time since the procedure itself had to be performed in standard fashion. The further increase in time mandated by providing the additional modality of ultrasound images (not routinely used during TIPS procedures at our institution) as well as the need for recruiting additional clinical 
personnel to perform the ultrasound imaging, and for such personnel to arrive at short notice before each procedure was not viewed as clinically acceptable.

The second reason for not employing 3D ultrasound was technical. Until recently, the only form of 3D ultrasound available to us acquired images using a manually swept system of image collection. Accurate 3D reconstruction of these images is difficult and contains inaccuracies. Such images could not provide a suitable gold standard for the studies outlined in this paper. More recently, true 3D ultrasound has become commercially available, but the units we have tested have not yet performed sufficiently well to be used in this study.

Our study possessed at least two limitations. The first was that our study included only 7 patients, of whom only 3 were studied during needle passage. Although we have acquired intraoperative images of many additional subjects, the study described in this paper required good quality, biplane images that depicted the same portion of the vasculature (or other identifiable intrahepatic structures) at two different times. We therefore had to exclude all cases in which monoplane images alone were obtained at one of the two time points, in which one of the sets of images was of poor quality, and in which clinical exigencies precluded acquiring a second set of biplane images for research purposes. It is rare to find patients with intrinsic, radiopaque structures within the liver, and we consider ourselves fortunate to have studied 3 such subjects during needle passage. The 7 patients described in the current paper represent all subjects acquired over the last 5 years whose images made them eligible for inclusion in the current study.

Please note, however, that our proposed approach to image-guided surgery does not have the same stringent image acquisition requirements. It is enormously easier to track a contrast-filled balloon automatically on biplane views than it is to repeatedly and manually define the same vessel branchpoints on pairs of projection views acquired at different times when present in different positions and imaged under differing imaging modalities. It is even more difficult to require the presence of individual gallstones visible by $\mathrm{x}$-ray or the presence of intrahepatic metallic clips inserted as a result of prior surgery, as mandated by the study of needle pressure described here, but certainly not required under our proposed approach to image guidance. Indeed, our proposed approach to image guidance requires manual definition of only a single target point on one view, with the corresponding point on the second view assisted by epipolar line drawing - it is not necessary for the purposes of surgical guidance to accurately define even a single vessel branchpoint on a pair of projection views! We have already developed automatic means of tracking the clinician's catheter from biplane views (Jolly et al.,2003) and of automatically tracking an implanted balloon. A complex, stereo display can be provided in real time (Maupu et al., 2005). The difficulty of obtaining a sufficient number of eligible patients is thus enormously greater when evaluating liver rigidity and motion and the basic assumptions of our proposed surgical approach, as performed under the current evaluative study, than in providing the image guidance methods themselves. A far larger number of patients are thus eligible for image guidance than were eligible for inclusion in the current study.

A second limitation was that we were able to define only a few 3D points within the liver for each patient and so could not provide a global grid of the liver. We therefore could not provide a dense map of liver deformation on the basis of the changing distances between hundreds of identifiable 3D points but were instead restricted to an analysis based upon the changing distances between a small number of 3D points. It should be noted, however, that all of the 3D points whose predicted and calculated positions were assessed in this study lay close to (and sometimes included) the actual clinical target point, or included even more distant points. If significant deformation occurred at the liver periphery our current study would not be able to 
detect such deformation, but point displacements in remote regions are not likely to be clinically relevant to the procedure in question.

The primary contribution of this paper is in its description of preliminary results suggesting that liver deformation during the TIPS procedure is less than $3 \mathrm{~mm}$ during both respiration and needle passage and that it appears possible to predict the location of an unseen target point within $3 \mathrm{~mm}$. This error in 3D point estimation is less than the $5 \mathrm{~mm}$ maximum error that our clinicians tell us would provide useful 3D image guidance. Whether the approach will prove useful during actual clinical practice has yet to be determined, however. Such determination will require a randomized study in which procedural time, number of needle passes, and complication rates are compared under the new and the old approaches. Nevertheless, the work described in this paper should be of relevance to all investigators interested in using 3D image guidance to improve the performance of TIPS and other x-ray guided procedures.

\section{ACKNOWLEDGEMENTS}

This work was supported by HL69808 NIH-HLB and CA124608 NIH-NCI..

\section{References}

Anand BS. Cirrhosis of liver. World J Med 1999;171:110-115.

Aylward S, Bullitt E. Initialization, noise, singularities and scale in height ridge traversal for tubular object centerline extraction. IEEE-TMI 2002;21:61-75.

Baert SAM, van de Kraats EB, van Walsum T, Viergever MA. Three-Dimensional Guide-Wire Reconstruction from Biplane Image Sequences for Integrated Display in 3-D Vasculature. IEEE TMI 2003;22:1252-1258.

Banares R, Casado M, Rodriques JM, Camunez F, Matilla A, Echenagusia A, Simo G, Piqueras B, Clemente G, Co E. Urgent transjugular intrahepatic portosystemic shunt for control of acute variceal bleeding. Am J Gastroenter 1998;93:75-79. [PubMed: 9448179]

Banovac F, Tang J, Xu S, Lindisch D, Chung HY, Levy EB, Chang T, McCullough MF, Yaniv Z, Wood BJ, Cleary K. Precision targeting of liver lesions using a novel electromagnetic navigation device in physiologic phantom and swine. Medical Physics 2005;32:2698-2705. [PubMed: 16193801]

Blackall, JM.; King, AP.; Penney, GP.; Adam, A.; Hawkes, DJ. Niessen, W.; Viergever, M. Proc MICCAI 2001. Vol. Vol 2208. LNCS Springer; 2001. A statistical model of respiratory motion and deformation of the liver; p. 1338-1340.

Blei AT. Portal hypertension and its complications. Curr Opin Gastroenterol 2007;23:275-282. [PubMed: 17414843]

Boctor EM, Fischer G, Choti MA, Fichtinger G, Taylor RH. A dual-armed robotic system for intraoperative ultrasound guided therapy hepatic ablative a prospective study. Proc. ICRA apos 2004;3:2517-2522.

Bosch J. Predictions from a hard liver. J Hepatol 2006;45:174-177. [PubMed: 16793168]

Boyer TD, Haskal ZJ. American Association for the Study of Liver Disease practice guidelines: The role of transjugular intrahepatic portosystemic shunt creation in the management of portal hypertension. J Vasc Interv Radiology 2005; 16:615-629.

Bricault I, DiMaio SM, Clatz O, Pujolo S, Vosburgh K, Kikinis R. Computer-assisted interventions on liver: Feasibility of the "anchor needle" technique for real-time targeting of lesions with respiratory motion. Surgetica. 2005Available at ftp://ftp-sop.inria.fr/epidaure/Publications/Clatz/Bricault_Surgetica.pdf

Bromley, PJ.; Kaufman, JA. Kaufman: Portal and Hepatic Veins. In: Kaufman, JA.; Lee, MJ., editors. Vascular and Interventional Radiology: The Requisites. Philadelphia PA: Elsivier, Inc; 2004. p. 377-406.

Clifford MA, Banovac F, Levy E, Cleary K. Assessment of Hepatic Motion Secondary to Respiration for Computer Assisted Interventions. Computer Aided Surgery 2002;7:291-299. [PubMed: 12582982] 
Faugeras, O. Three-Dimensional Computer Vision. A Geometric Viewpoint MIT Press; 1996.

Haskal ZJ, Martin L, Cardella F, Cole PE, Drooz A, Grassi C, McCowan TC, Meranze SG, Neithhamer CD, Oglevie SB, Roberts AC, Sacks D, Silverstein MI, Swan TL, Towbin RB, Lewis CA. Quality improvement guidelines for transjugular intrahepatic portosystemic shunts. J Vasc Interv Radiol 2001;12:131-136. [PubMed: 11265875]

Hassoun Z, Pomier-Layrargues GP. The transjugular intrahepatic portosystemic shunt in the treatment of portal hypertension. Eur J Gastroenterol Hepatol 2004;16:1-4. [PubMed: 15095845]

Jolly B, Van Horn M, Aylward S, Bullitt E. Bullitt Needle tracking and detection in the TIPS endovascular procedure. MICCAI 2003; Lecture Notes in Computer Science 2003;2878:953-954.

Kazemi F, Kettaneh A, N'kontchou G, Pinto E, Ganne-Carrie N, Trinchet JC. Liver stiffness measurement selects patients with cirrhosis at risk of bearing large oesophageal varices. J Hepatol 2006;45:230 235. [PubMed: 16797100]

Kee ST, Rhee JS, Butts K, Daniel B, Pauly J, Kerr A, O’Sullivan GJ, Sze DY, Razavi MK, Semba CP, Herfkens RJ, Dake MD. MR-guided transjugular portosystemic shunt placement in a swine model. J Vasc Interv Radiol 1999;10:529-535. [PubMed: 10357476]

King AP, Blackall JM, Penney GP, Hawkes DJ. Tracking liver motion using 3-D ultrasound and a surface based statistical shape model. Proc Mathematical Methods in Biomedical Image Analysis MMBIA 2001. IEEE Computer Society 2001:145-152.

LaBerge JM, Ring EJ, Gordon RL, Lake JR, Doherty MM, Somberg KA, Roberts JP, Ascher NL. Creation of transjugular intrahepatic portosystemic shunts with the Wallstent endoprosthesis: Results in 100 patients. Interventional Radiology 1993;187:413-420.

Maupu D, Van Horn MH, Weeks S, Bullitt E. 3D interactive stereo visualization for the TIPS procedure. IEEE Computer Graphics and Applications 2005;25:67-71. [PubMed: 16209172]

Miga MI, Cash DM, Cao Z, Galloway RL, Dawant B, Chapman WC. Intraoperative registration of the liver for image-guided surgery using laser range scanning and deformable models, Medical Imaging 2003: Visualization, Image-guided Procedures, and Display. Proc. of the SPIE 2003;Vol. 5029:350359.

Pathak AC, Van Horn M, Weeks S, Bullitt E. Comparison of simultaneous and sequential two-view registration for 3D/2D registration of vascular images. MICCAI 2005;3750:239-246. [PubMed: 16685965]LNCS

Rohlfing, T.; Maurer, CR.; O’Dell, WG.; Zong, J. Proceedings of SPIE. Vol. vol 4319. Bellingham, WA:: SPIE; 2001. Modeling liver motion and deformation during the respiratory cycle using intensitybased free-form registration of gated MR images. Medical Imaging: Visualization, Display, and Image-Guided Procedures (2001); p. 337-348.

Rose SC, Behling C, Roberts AC, Pretorius DH, Nelson TR, Kinney TB, Masliah E, Hassanein TI. Main portal vein access in transjugular intrahepatic portosystemic shunt procedures: Use of threedimensional ultrasound to ensure safey. J Vasc Interv Radiol 2002;13:267-273. [PubMed: 11875086]

Rose SC, Pretorius DH, Nelson TR, Kinney TB, Huynh TV, Roberts AC, Valji K, D’ Agostino HR, Oglevie SB, James GM, Hassanein TI, Hart ME, Orloff MJ. Adjunctive 3D US for achieving portal vein access during transjugular intrahepatic portosystemic shunt procedures. JVIR 2000;11:611-621. [PubMed: 10834493]

Saxon RR, Keller FS. Technical aspects of accessing the portal vein during the TIPS procedure. J Vasc Interv Radiol 1997;8:733-744. [PubMed: 9314363]

Tokuda J, Hirano M, Tsukamoto T, Dohi T, Hata N. Real-time organ motion tracking and fast image registration system for MRI-guided surgery. Systems and Computers in Japan 2006;37(1):83-92.

Sze DY, Strobel N, Fahrig R, Moore T, Busque S, Frisoli JK. Transjugular intrahepatic portosystemic shunt creation in a polycystic liver faciliated by hybrid cross-sectional/angiographic imaging. J Vasc Interv. Radiol 2006;17:711-715. [PubMed: 16614155]

Venkatraman V, Horn MVan, Weeks S, Bullitt E. Liver Motion Due to Needle Pressure, Cardiac and Respiratory Motion During the TIPS Procedure. MICCAI 2004; Lecture Notes in Computer Science 2004;3217:66-72.

Virmani S, Ryu R, Sato K, Lewandowski R, Kulik L, Mulcahy M, Larson A, Salem R, Omary R. Effect of c-arm angiographic CT on transcatheter arterial chemoembolization of liver tumors. JVIR 18:1305-1309. 

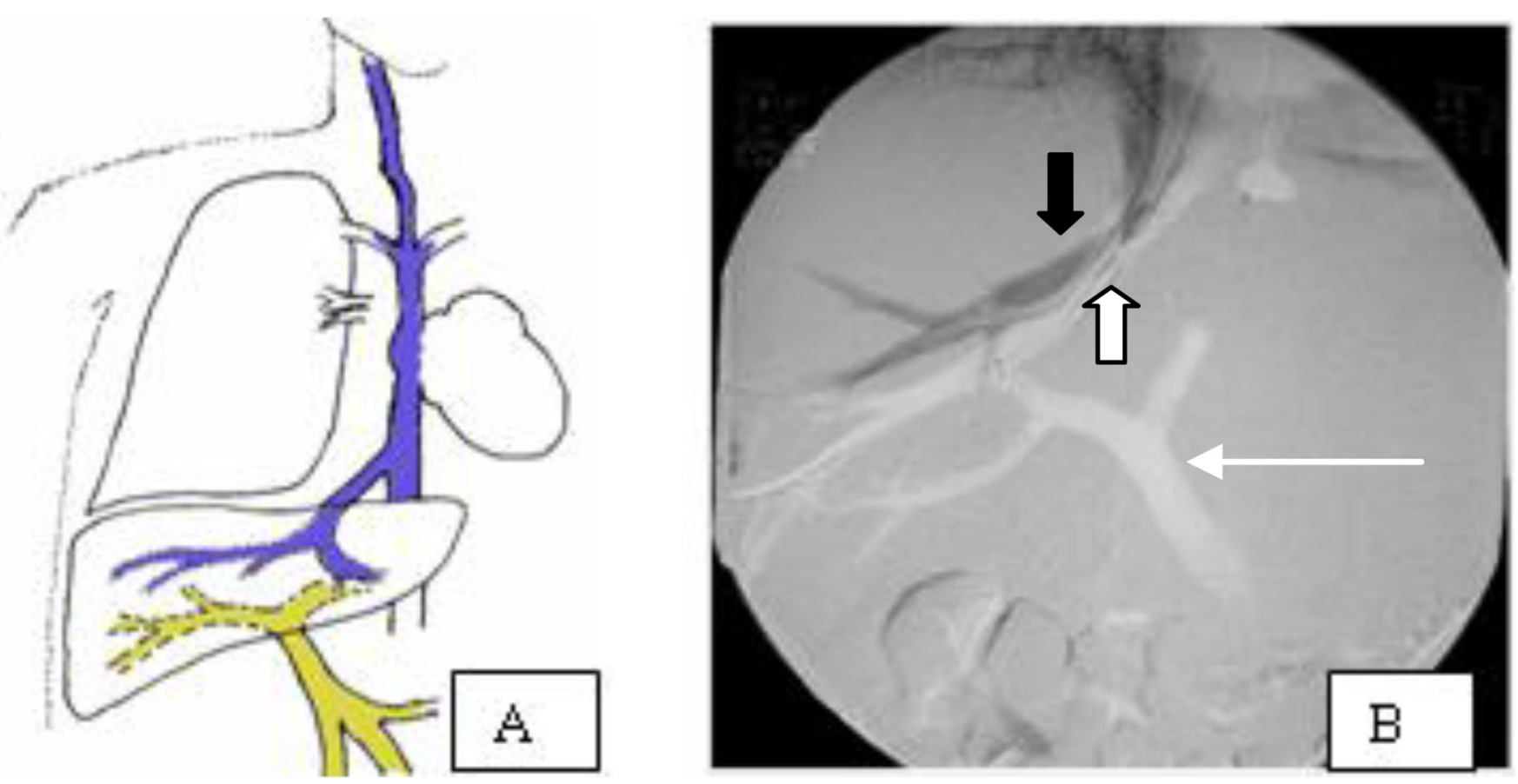

Fig. 1.

Overview of the procedure. A: Drawing of the liver, chest and hepatic venous system (blue) and the portal venous system (gold). The goal of the TIPS procedure is to create an artificial connection between the hepatic and portal veins. B. X-ray image with opacification of hepatic vein with injected contrast material (dark) with overlay of a static, preacquired, subtracted image of the portal and hepatic venous systems (white) prior to needle insertion. Thin white arrow points to the portal veins and white arrowhead points to hepatic veins at time 1 , and black arrowhead points to hepatic veins at time 2 . Note the superior translation of the hepatic veins (black at time 2) due to respiratory motion between the two image acquisitions. 

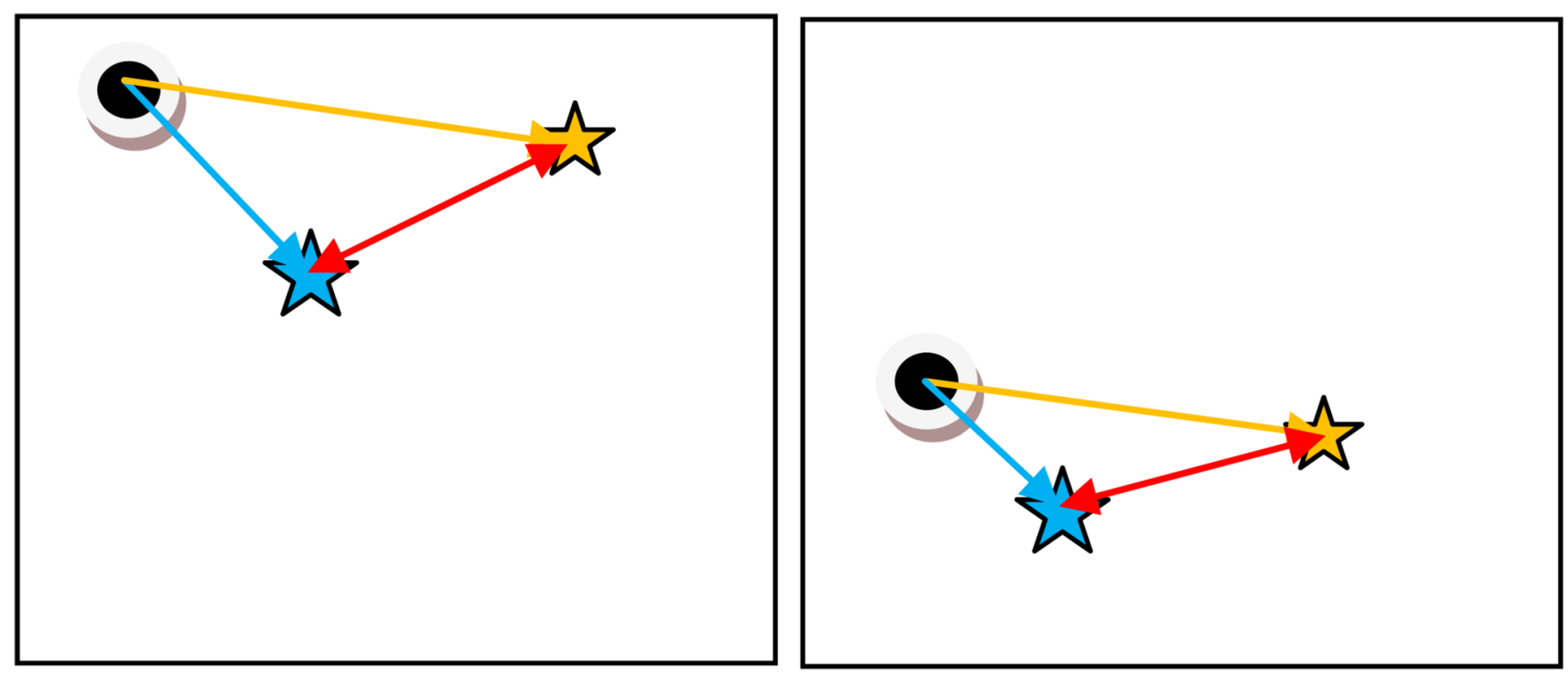

Fig. 2.

Simplified overview of the approach illustrated by a single AP image at time 1 (left) and time 2 (right). Black ball = projection of the opacified balloon. Gold and blue stars $=$ projections of "point blue" and "point gold" Gold arrow = vector between balloon and "point gold". Blue arrow $=$ vector between balloon and "point blue". Red arrow = distance between points blue and gold. The opacified balloon (black) has moved downward at time 2 . If there is no liver deformation the distances between all three points should remain constant at times 1 and 2 (the lengths of the red, gold, and blue arrows should remain constant). If it is possible to use the opacified balloon to track the new position of "point blue" at time 2, the blue vector (determined at time 1) when added to the new balloon position at time 2 should correctly point to "point blue" at time 2. Similarly, the new position of "point gold" should be predicted by adding the gold vector defined at time 1 to the new balloon position at time 2. Errors induced by liver rotation or deformation can be estimated by comparing the predicted to the actual locations of points blue and gold. This figure illustrates a simplified approach in 2D; our study employed all vector direction and length calculations in $3 \mathrm{D}$. 


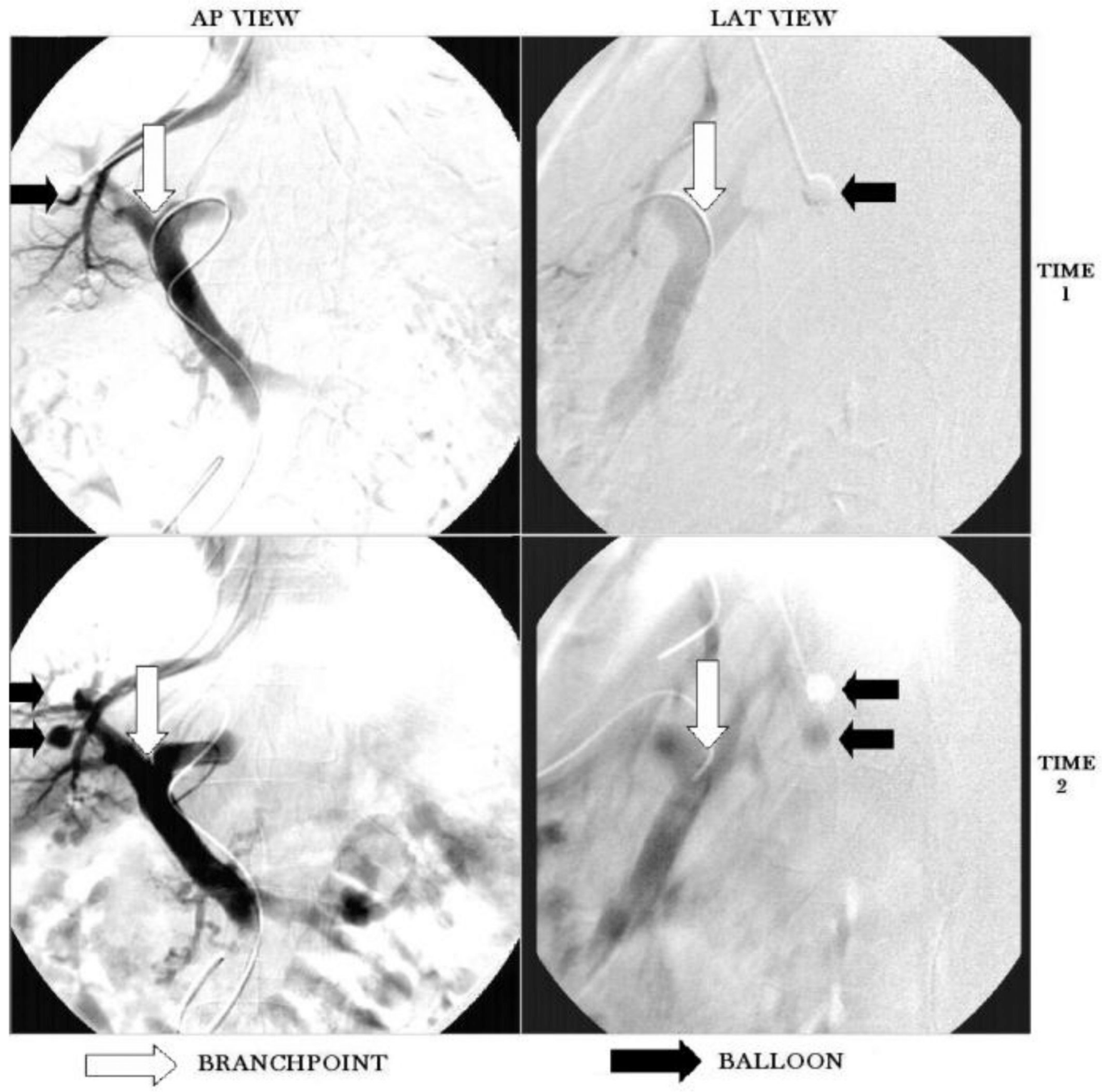

Figure 3.

AP (left) and lateral (right) angiograms obtained at time 1 (top row) and subtracted images obtained at time 2 (bottom row). The solid black arrows point to the balloon in each image. The white arrows point to one of the several identified vessel branchpoints. Note that on the subtracted images at time 2 that both the original balloon position is evident (white disc, black arrow) as well as the new balloon position (dark disc, black arrow). There is obvious displacement of the balloon as well as of the identified branchpoint (white arrow) between times 1 and 2. 


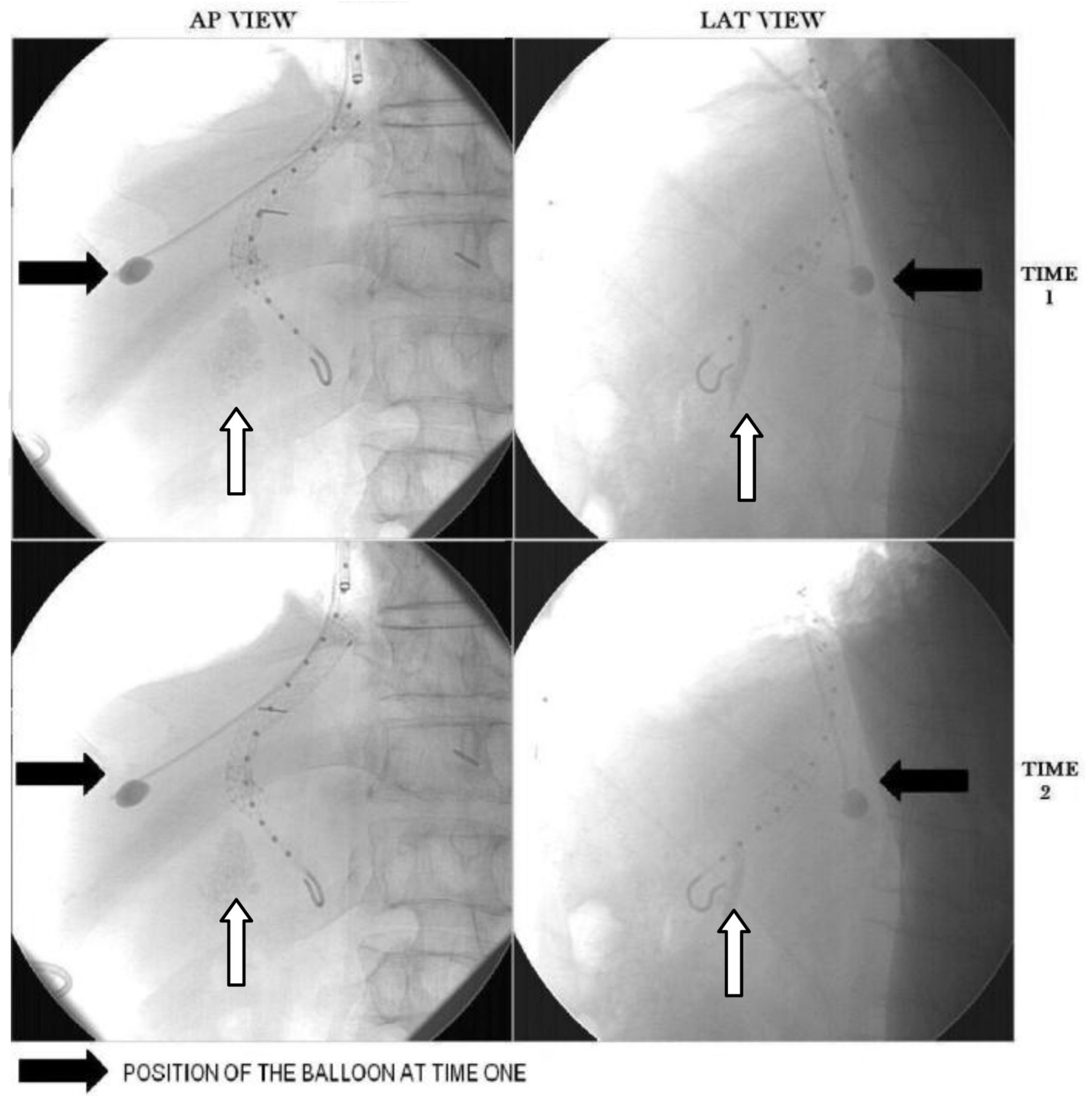

Figure 4.

AP (left) and lateral (right) views of balloon, catheter within the deployed TIPS stent, and opacified gall bladder obtained at times 1 (top row) and 2 (bottom row). The black arrow shows the position of the balloon at time 1 . The white arrow points to the partially opacified gall bladder. The catheter within the TIPS stent is the curved line with multiple dots along its length. There is downward motion of the balloon, gallbladder, and stent between times 1 and 2 . 


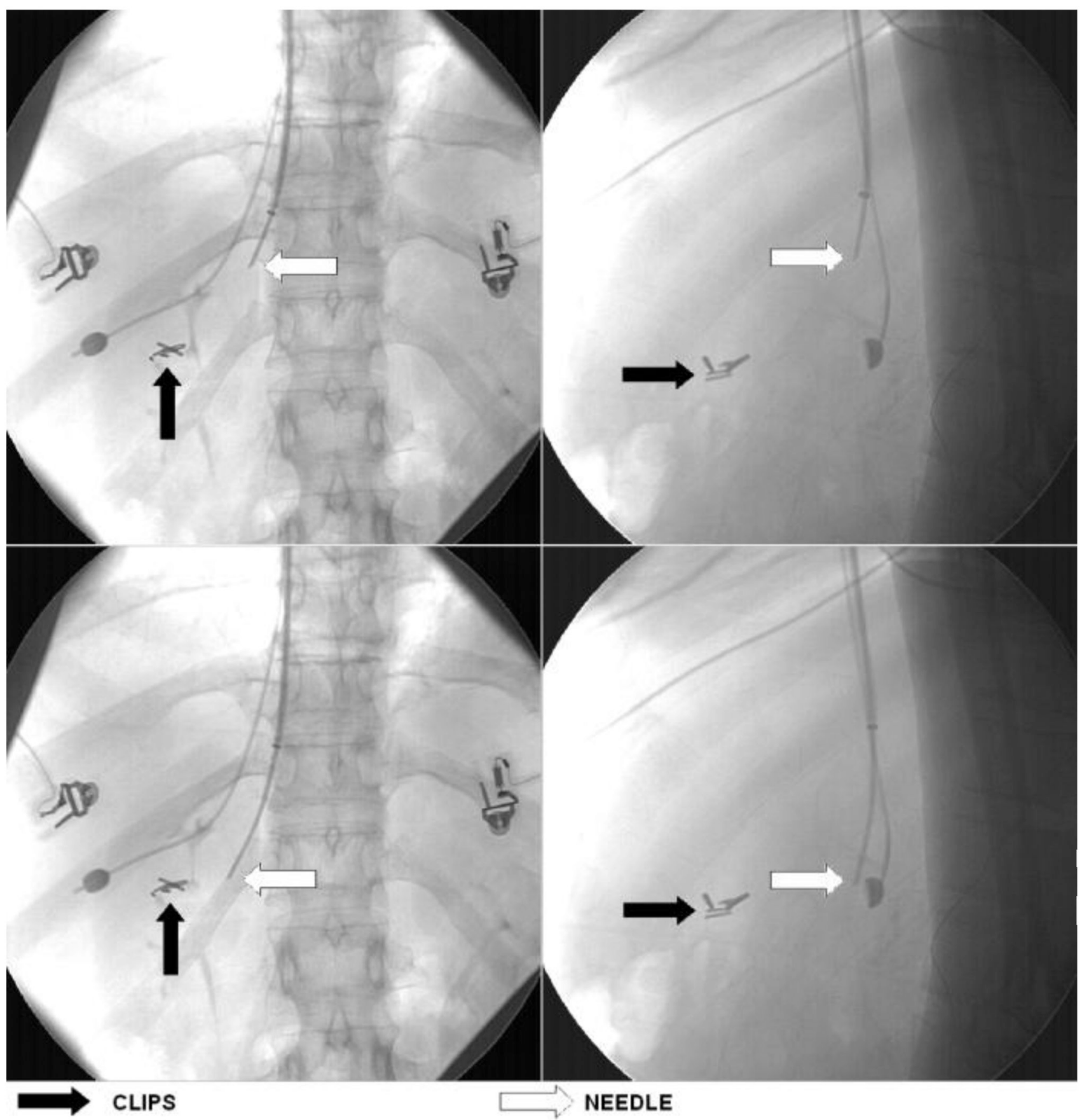

Fig. 5.

AP (left) and Lat (right) images of the needle push study for patient with clips (black arrow), needle (white arrow) and balloon at time 1 (top) and time 2 (bottom). 


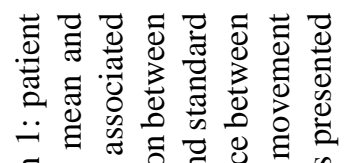

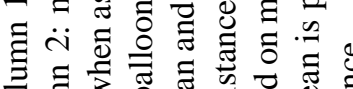

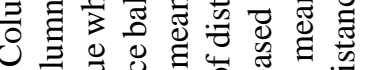

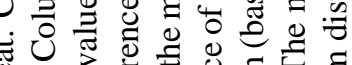

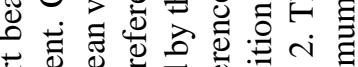

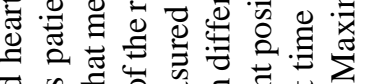

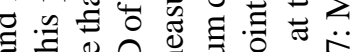

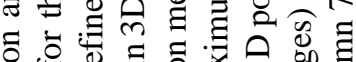

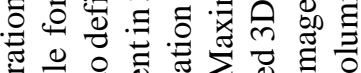

: $\frac{0}{3}$ 웧

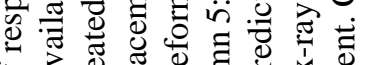

पै

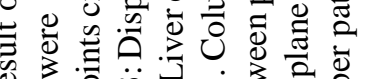

巳

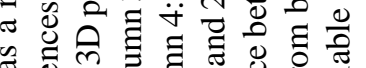

๘

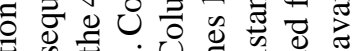

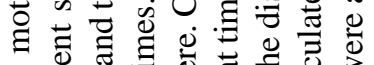

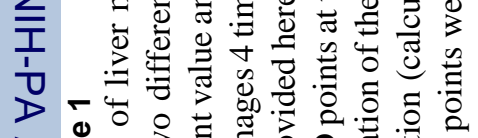

文

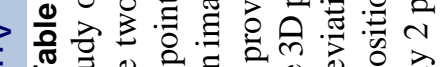

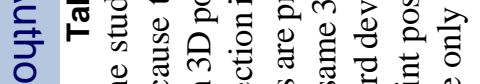

可

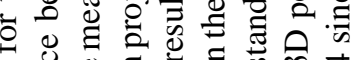

.

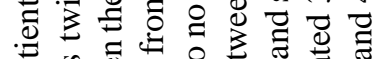

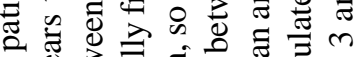

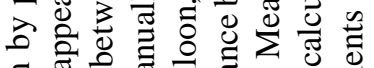

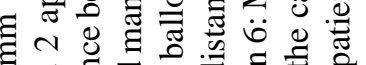

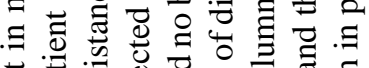

$\rightarrow=$ :

这

ฮั่

‡

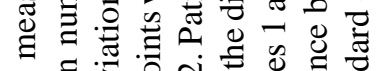

¿.

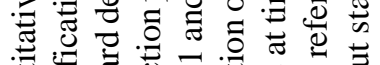

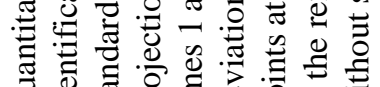

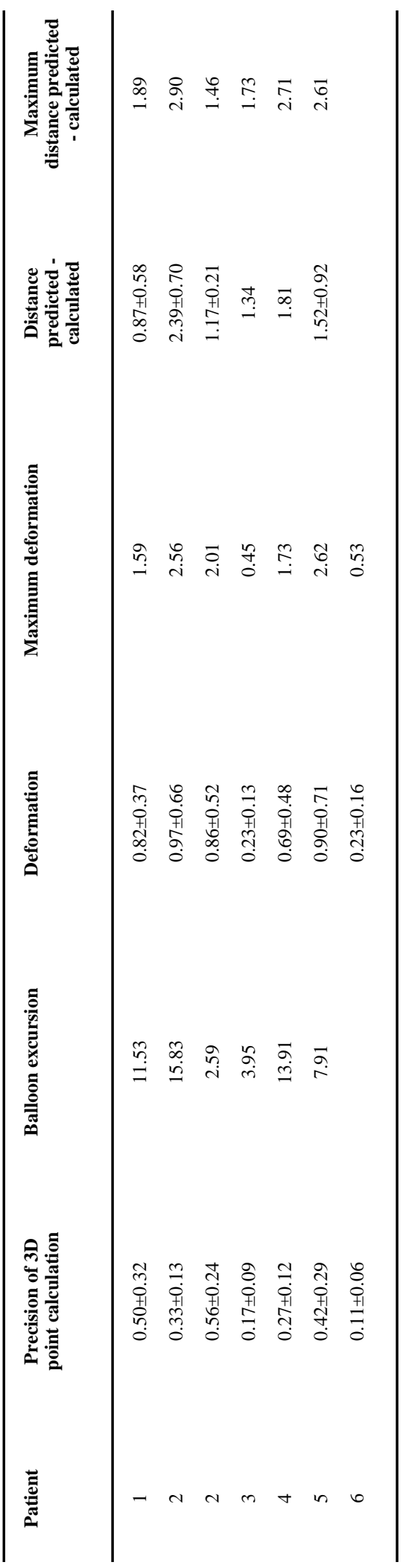

곻

Med Image Anal. Author manuscript; available in PMC 2010 June 1. 


\section{Table 2}

Needle push study (mm). Column 1: Patient ID. Column 2: mean and standard deviation of the precision of point selection. Column 3: 3D displacement of the reference balloon between times 1 and 2. Column 4: 3D displacement of the needle tip between time 1 and time 2. Column 5: Mean and standard deviation of the distance between predicted $3 \mathrm{D}$ point position and the calculated $3 \mathrm{D}$ point position. Two values are given for patient 5 since two points were picked on the gallbladder.

\begin{tabular}{ccccc}
\hline Patient & $\begin{array}{c}\text { Precision of 3D } \\
\text { point calculation }\end{array}$ & Balloon excursion & Needle push & Distance predicted -calculated \\
\hline 2 & $0.38 \pm 0.18$ & 7.25 & 19.18 & 2.12 \\
5 & $0.44 \pm 0.16$ & 7.27 & 50.30 & $1.76-1.35$ \\
7 & $0.29 \pm 0.10$ & 1.28 & 31.18 & 0.69 \\
\hline
\end{tabular}

